\title{
La Responsabilidad Social Corporativa en los estudios universitarios de comunicación ${ }^{1}$
}

\section{The Corporate Social Responsibility in undergraduate communication studies}

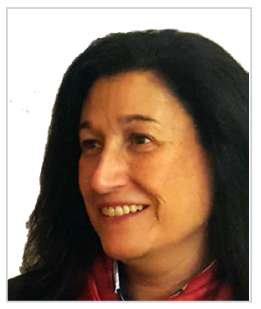

María Teresa García Nieto. Profesora Titular de Universidad, ha sido Vicedecana de la Facultad de Ciencias de la Información de la Universidad Complutense de Madrid y Directora del título de Comunicación Audiovisual en el Centro de Estudios Superiores Felipe II de la misma universidad. Cuenta con el reconocimiento de la actividad investigadora de la CNEAI. Compatibiliza sus tareas de docencia e investigación con trabajos de consultoría y asesoría de comunicación. La profesora García Nieto es investigadora principal de diversos proyectos de investigación y de innovación educativa. Ha dirigido numerosas tesis doctorales, dos de ellas con premio extraordinario, además de investigaciones doctorales y trabajos fin de máster en varias universidades, tanto nacionales como internacionales. Forma parte de numerosos comités científicos de congresos internacionales y de los comités editoriales de varias revistas científicas indexadas. Es miembro de los Comités de Evaluación de Títulos Universitarios, del área de Ciencias Sociales, de las Agencias para la Calidad del Sistema Universitario en varias comunidades autónomas. Universidad Complutense de Madrid, España xyz@ccinf.ucm.es

ORCID: 0000-0002-6235-511X

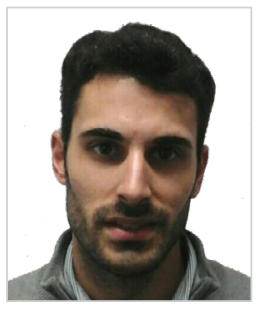

Julio Manuel Gil Rodríguez. Máster en Comunicación de las Organizaciones por la Universidad Complutense de Madrid, posee el Grado en Administración y Dirección de Empresas por la Universidad de Extremadura. Universidad Complutense de Madrid, España juliogil@ucm.es ORCID: 0000-0002-9561-4114

Recibido: 02/11/2018 - Aceptado: 18/11/2018

Received:02/11/2018 - Accepted: 18/11/2018

\section{Resumen:}

La formación de nuestros futuros profesionales en materia de Responsabilidad Social ha adquirido una gran importancia en los últimos años. Los gestores deben ser conscientes de la condición de sus organizaciones como entidades promotoras del desarrollo social.

\section{Abstract:}

Training of future professionals on the issue of social responsibility has acquired increased importance in recent years. Managers and corporative leaders should be aware of the role of organizations in social development. This research analyses the current state of

1 Esta investigación se ha desarrollado en el marco de los Proyectos Innova-Docencia “Universidad y sociedad: comunicación e integración en empresas e instituciones públicas y organizaciones no lucrativas”. Refs. 281, 176 y 193 (2016-2019) de la Universidad Complutense de Madrid.

Cómo citar este artículo:

García Nieto, M. T.; Gil Rodríguez, J. M. (2018). La Responsabilidad Social Corporativa en los estudios universitarios de comunicación. Doxa Comunicación, 27, pp. 387-418. 
La presente investigación analiza el estado actual de la formación sobre Responsabilidad Social Corporativa en la oferta académica en los estudios de grado en las universidades españolas, públicas y privadas. Nuestra investigación se centra en el ámbito de la comunicación, como uno de los pilares fundamentales vinculados a la gestión de la RSC. Basada en la información obtenida de entrevistas en profundidad, realizamos un análisis de las guías docentes de las materias sobre RSC en los grados de comunicación y disciplinas afines. Palabras clave:

Responsabilidad social corporativa; responsabilidad social universitaria; educación universitaria; comunicación; formación en responsabilidad social.
CSR, as a subject in undergraduate curricula in public and private universities. Our research focuses on the field of communication and its linkage to SCR management. Based on the results of indepth interviews, we carry out a documentary analysis of SCR subject syllabi of undergraduate studies in communication and related disciplines.

\section{Keywords:}

Corporate social responsibility; university social responsability; university education; communication; social responsibility training.

\section{Introducción. La responsabilidad social en la universidad}

Una organización es socialmente responsable cuando tiene en cuenta las necesidades, preocupaciones e intereses de los grupos sociales con los que se relaciona en el proceso de toma de decisiones, en sus políticas y planes estratégicos, en su gestión, sus procedimientos, sus conductas y su comunicación. Y cuando asume sus consecuencias. Tanto con los públicos contemporáneos como con los futuros. Con personas y grupos cuya vinculación deriva de las actividades propias de la organización, o con aquellos que tienen una vinculación extraordinaria, con personas ajenas a la organización. Ya se vean afectados por ella a corto, medio o largo plazo; cerca o lejos (García Nieto, 2012: 94).

La Responsabilidad Social es una estrategia de gerencia ética e inteligente de los impactos que genera la organización en su entorno humano, social y natural. Gerencia ética: Todos los potenciales afectados por la actividad de la organización deben de retirar los mayores beneficios y los menores daños de ella. La organización debe servir al mundo, y no sólo servirse del mundo. Gerencia inteligente: La gestión responsable de los impactos de la organización debe de retornar en beneficios para la organización, cada vez que sea posible, para que la Responsabilidad Social de la organización sea una política sostenible y eficiente. Al ser socialmente responsable, la organización se desarrolla mejor en un entorno mejor. (Vallaeys, 2013: 3).

La responsabilidad social se ha convertido en una exigencia cada vez mayor para las organizaciones en nuestra sociedad. Y las universidades españolas no han sido ajenas a este fenómeno, convirtiéndose en pioneras de este tipo de prácticas socialmente responsables. Hace ya más de una década, el 88,6\% de las organizaciones universitarias españolas asumía entre sus funciones actividades solidarias propias de la responsabilidad social corporativa (Arias Careaga y Simón Ruiz, 2004). Vivimos en un mundo cada día más consciente de la necesidad de establecer las bases de una sociedad instruida, guiada por profesionales socialmente responsables, con valores éticos y transparentes, y con el propósito de un futuro sostenible.

En España, el primer proyecto educativo en el ámbito universitario europeo, que integró en sus planes de estudios una asignatura obligatoria con el objetivo de formar alumnos en responsabilidad social, fue en 1993, por iniciativa de la Universidad Francisco de Vitoria (De la Calle Maldonado, 2011). 
El Consejo Estatal de Responsabilidad Social de las Empresas (CERSE), órgano asesor y consultivo del Gobierno de España, actualmente adscrito al Ministerio de Empleo y Seguridad Social, dependiente de la Dirección General del Trabajo Autónomo, de la Economía Social y de la Responsabilidad Social de las Empresas, incluye, entre sus grupos de trabajo, uno específico sobre "Educación, Formación y Divulgación de la RSE”.

En octubre de 2014, el Consejo de Ministros aprobaba la Estrategia Española de Responsabilidad Social de las Empresas 2014-2020, respondiendo a las recomendaciones de la Unión Europea en su Estrategia Renovada sobre Responsabilidad Social de las Empresas, con el objetivo de "avanzar hacia una sociedad y una economía más competitiva, productiva, sostenible e integradora” (Ministerio de Empleo y Seguridad Social -MESS-, 2015:1).

Este documento que pretende consensuar y conceptualizar la importancia de la RSC, en las empresas, administraciones públicas, y la sociedad en general, incluye entre sus objetivos "Difundir los valores de la RSE en el conjunto de la sociedad" (MESS, 2015: 30). Además, entre sus líneas de actuación, se especifica la "Integración de la RSE en la educación, la formación y en la investigación” (MESS, 2015: 31).Y, entre las medidas propuestas, se indica "Potenciar el estudio de la RSE tanto en las instituciones educativas para la formación profesional como en las universidades para la enseñanza universitaria, así como en los centros de investigación" (MESS, 2015: 37). Todo ello partiendo de la siguiente consideración:

La educación en el valor del comportamiento responsable, la formación especializada en técnicas de gestión socialmente responsable y la investigación orientada a la aplicación efectiva de sus criterios, son pilares fundamentales para acelerar cambios en la forma de pensar y el actuar sostenible de las actuales y futuras generaciones (MESS, 2015: 36).

Y, como es sabido, las universidades tienen la responsabilidad de:

- Ofrecer formación de alto nivel en materia científica, profesional, humanística, artística y técnica.

- Contribuir: a la competitividad económica y al desarrollo humano sostenible.

- Promover la generación, desarrollo y difusión del conocimiento en todas sus formas.

- Contribuir a la preservación de la cultura nacional, y desarrollar las actitudes y valores.

- Formar a personas responsables, con conciencia ética y solidaria, reflexivas, innovadoras, críticas, capaces de mejorar la calidad de vida, consolidar el respeto al medio ambiente, a las instituciones del país y a la vigencia del orden democrático (Díaz de Iparraguirre, 2008: 4).

Además, como señala el Equipo coordinador Universidad Construye País, (Fernández, Delpiano, y De Ferari, 2006), la mayor contribución que los centros de educación superior pueden ofrecer a la sociedad es, precisamente, la formación de profesionales cualificados, pero comprometidos, conscientes y capaces de valorar las consecuencias de su ejercicio profesional.

Vallaeys, De la Cruz y Sasia (2009) resumen en cuatro procesos interdependientes la autorregulación de la gestión corporativa en relación con las definiciones y prácticas de la responsabilidad social, de tal manera que estos procesos confirman la naturaleza social de la organización y la ayudan a situarse en la sociedad. Los procesos referidos son:

- El buen gobierno. La buena gobernanza consiste en cumplir de manera coherente y congruente con la misión propia de la organización, en implementar un código ético y contar con un comité autónomo encargado de promover y vigilar su cumplimiento. Se trata no sólo de asegurar la observancia de las normas legales nacionales, sino también de las 
pautas internacionales más exigentes de carácter laboral, social y medioambiental. Se trata de promover y conseguir un buen clima laboral, luchar contra la inequidad, las desigualdades y la discriminación. Se trata de salvaguardar los derechos humanos de todas las personas e informar de manera honesta y transparente de los resultados de la gestión de la organización. Un buen gobierno promueve la RSC de una organización liberándola de todo atisbo de corrupción.

- El diálogo y la interlocución con los diferentes públicos de la organización, a los que debe rendir cuentas. Vallaeys, De la Cruz y Sasia recurren al modelo de los stakeholders (grupos de interés) de Freeman y Reed (1983), considerando la organización como una estructura abierta en la cual concurren los riesgos e intereses de los diversos actores internos o externos a la organización, vinculados o no con ella, y que se puedan ver afectados por su actividad, tengan o no la capacidad de afectarla. La organización debe responder, de manera satisfactoria, a los requerimientos de los diferentes grupos de interés, estableciendo con ellos vínculos y relaciones transparentes y simétricas, informándoles de los resultados de los acuerdos adoptados. El diálogo con los públicos constituye un proceso de regulación socialmente responsable evitando el riesgo del egocentrismo en la organización.

- La gestión de los impactos sociales y medioambientales. La filosofía gerencial de la responsabilidad social implica hacer consciente a la organización de las consecuencias y efectos que sus decisiones, sus planes estratégicos y sus comportamientos, puedan provocar en las personas y grupos sociales, así como en el medioambiente. La organización debe tomar conciencia de su impacto en el entorno, tanto interno como externo, promoviendo el desarrollo sostenible. Así, la responsabilidad social constituye una política gerencial de la organización que la hace permeable y empática.

- Estos autores añaden que, para practicar una gestión socialmente responsable orientada al logro del desarrollo social y medioambiental sostenible, es preciso avanzar desde una lógica reactiva hacia una lógica proactiva de implicación en la solución de los problemas sociales del entorno de la organización. Este cambio debe basarse, en cualquier caso, en el cumplimiento con la misión, los principios y valores corporativos, en la gestión de los impactos y en la comunicación y participación efectiva de los diferentes grupos de interés. La proactividad hacia la gestión socialmente responsable propicia la creación de un "capital social" de la organización, así como la posibilidad de llegar a acuerdos para tratar de manera conjunta los problemas sociales. Y esta proactividad socialmente responsable, fundamentada en la empatía social de la organización, en una filantropía desinteresada, le permite anticipar los riesgos que afectan a la organización. En este sentido, la responsabilidad social se convierte en un una vacuna frente al egoísmo corporativo.

- Siguiendo a los citados autores, Vallaeys, De la Cruz y Sasia (2009), la responsabilidad social de las universidades, sin embargo, ha de diferenciarse de la responsabilidad social corporativa o empresarial, en tanto que la responsabilidad social universitaria debe definirse fundamentalmente por la gestión de sus impactos en el entorno. Los cuales, pueden agruparse a tenor de cuatro criterios.

- Los impactos de funcionamiento derivados de la actividad propia de la organización sobre los públicos con los que la universidad se relaciona directamente, como el personal docente y el de administración y servicios, los estudiantes, o la comunidad en la que la universidad se ubica. Se incluyen aquí, también, los impactos que se producen como consecuencia de la práctica de sus funciones, tales como la contaminación. La huella social y ecológica de la organización universitaria.

- Los impactos educativos. El cumplimiento de la misión didáctica de la universidad debe generar un impacto necesario y pretendido en la formación de los jóvenes y futuros profesionales. Este impacto pedagógico propicia el aprendizaje de los estudiantes fomentando su capacidad de analizar, entender e interpretar el mundo, para saber comportarse en 
él, y desarrollar su competencia crítica de manera que puedan valorar las cuestiones importantes de su vida. Estos impactos afectan, también, al aprendizaje de los códigos éticos y la deontología profesional, así como de la función social de cada disciplina. En este sentido, la universidad socialmente responsable se cuestiona sobre el tipo de personas que se propone formar, planificando adecuadamente las enseñanzas para lograr profesionales éticos, íntegros y responsables, quienes con el ejercicio de sus carreras contribuirán a la construcción de una sociedad más justa y sostenible.

- Los impactos cognitivos y epistemológicos. La universidad no sólo tiene una función pedagógica, también tiene la misión de producir y transferir a la sociedad el conocimiento científico, tecnológico, social y humanístico.

- Los impactos sociales: La universidad, como cualquier organización, es también una entidad social, un actor social que interactúa con su entorno para contribuir al desarrollo y la sostenibilidad de los grupos sociales externos a la universidad con los cuales se relaciona. La universidad debe ser un agente activo partícipe en el progreso y el desarrollo de un país.

El Grupo de investigación Ingeniería y Gestión responsable de la Universidad de Burgos sintetiza el concepto de responsabilidad social universitaria del siguiente modo:

La RSU es la capacidad de la Universidad de aplicar un conjunto de principios y valores en la realización de sus funciones básicas: (1) formación académica y pedagógica, (2) investigación y difusión, (3) gestión de la organización y (4) participación social, mediante la creación de canales de comunicación y participación para responder a las demandas de sus grupos de interés (González Alcántara et al. 2015: 7).

Y la UNED (Universidad Nacional de Educación a Distancia), que presentó su primer Informe Diagnóstico de la RS hace ya diez años, lo deja claro en su página web:

Para la UNED, 'responsabilidad social' significa ofertar servicios educativos y transferencia de conocimientos siguiendo principios de ética, buen gobierno, respeto al medioambiente, compromiso social y promoción de valores ciudadanos; responsabilizarse, por lo tanto, de las consecuencias y los impactos que se derivan de sus acciones (UNED, 2018).

Parece evidente, por ende, que la responsabilidad universitaria no puede interpretarse en los mismos términos que la responsabilidad social corporativa o empresarial. Ni siquiera en el caso de las universidades privadas. Pues, si bien es cierto que todas las universidades, públicas y privadas, deben garantizar su sostenibilidad económica, ésta no puede ser, de ningún modo, una prioridad frente a las responsabilidades éticas y sociales de este tipo de organizaciones educativas. No parece conveniente, ni adecuado, por tanto, sustentar nuestra investigación en fuentes teóricas propias de las ciencias empresariales, como la teoría de Archie Carroll $(1991,1998)$ quien propone una definición general de la responsabilidad social empresarial estructurada en cuatro categorías: la económica, la legal, la ética y la discrecional, o filantrópica, pero para quien las responsabilidades económicas constituyen la base de los compromisos corporativos.

En este mismo sentido, en el que prima claramente el interés de la empresa, se desarrollan los Principios para la Educación de la Gestión Responsable (PRME), iniciativa que data de 2007, surgida con el apoyo de las Naciones Unidas. PRME constituye una plataforma formada por instituciones de educación superior de todo el mundo, directamente vinculadas con la formación en materia de gestión, a través de las escuelas de administración y negocios, con el objetivo de "elevar el perfil de la sostenibilidad en las escuelas de todo el mundo y para equipar a los estudiantes de negocios de hoy con la comprensión y la capacidad de generar cambios en el futuro" (PRME, 2018). 
Como instituciones de educación superior involucradas en el desarrollo de gerentes actuales y futuros, declaramos nuestra voluntad de progresar en la implementación, dentro de nuestra institución, de los siguientes Principios, comenzando con aquellos que son más relevantes para nuestras capacidades y misión...:

Principio 1 I Propósito: Desarrollaremos las capacidades de los estudiantes para ser futuros generadores de valor sostenible para las empresas y la sociedad en general y para trabajar por una economía global inclusiva y sostenible.

Principio 2 । Valores: Incorporaremos en nuestras actividades académicas, planes de estudio y prácticas organizacionales los valores de la responsabilidad social global como se muestra en iniciativas internacionales como el Pacto Mundial de las Naciones Unidas.

Principio 3 । Método: Crearemos marcos educativos, materiales, procesos y entornos que permitan experiencias de aprendizaje efectivas para un liderazgo responsable.

Principio 4 I Investigación: participaremos en investigaciones conceptuales y empíricas que avancen nuestra comprensión sobre el papel, la dinámica y el impacto de las corporaciones en la creación de un valor social, ambiental y económico sostenible.

Principio 5 | Asociación: interactuaremos con los gerentes de las corporaciones comerciales para ampliar nuestro conocimiento de sus desafíos en el cumplimiento de las responsabilidades sociales y ambientales y para explorar enfoques conjuntos efectivos para enfrentar estos desafíos.

Principio 6 | Diálogo : Facilitaremos y apoyaremos el diálogo y el debate entre educadores, estudiantes, empresas, gobiernos, consumidores, medios de comunicación, organizaciones de la sociedad civil y otros grupos interesados y partes interesadas sobre temas críticos relacionados con la responsabilidad social global y la sostenibilidad (PRME).

Estos principios, PRME, son recogidos expresamente por el citado Grupo de Trabajo sobre Educación, Formación y Divulgación de la RSE del Consejo Estatal de Responsabilidad Social de las Empresas (CERSE), del Ministerio de Empleo y Seguridad Social del Gobierno de España, en su documento "La responsabilidad social de las empresas (RSE), el desarrollo sostenible y el sistema de educación y Formación” (2010).

Pero, como hemos señalado, el enfoque de este discurso se orienta fundamentalmente hacia la propia empresa como referente de la responsabilidad social. Es decir, su foco de atención es la empresa como entidad generadora de riqueza y sostenibilidad económica y medioambiental. Y de esta manera, el punto de mira del proceso de planificación de la responsabilidad social corporativa es la propia organización, la misma empresa. Hablamos de responsabilidad social empresarial (RSE).

Este punto de vista fundamentado en la empresa como negocio, no nos parece el más adecuado para cimentar nuestra investigación. Consideramos mucho más oportuno acudir a los fundamentos teóricos de los campos científicos no empresariales, no economicistas, como el propio de las relaciones con los públicos, atendiendo a la teoría de los stakeholders de Freeman y Reed (1983) o al enfoque de las relaciones públicas socialmente responsables de Grunig (Grunig y Hunt, 1984; Grunig, 1992).

Para Freeman, la responsabilidad social de las organizaciones consiste en integrar los intereses de todos los grupos con los que éstas se relacionan, priorizando el objetivo de la sostenibilidad de sus actividades e insistiendo en la importancia de la planificación a largo plazo, pero sin olvidar los resultados previstos a corto plazo. Es preciso, por tanto planificar las relaciones con los stakeholders, o grupos de interés, integrando las diferentes visiones de cada uno de ellos. Ya se entienda a los stakeholders en sentido restringido, incluyendo a cualquier persona o grupo con quien se relaciona la organización, y de 
quienes ésta depende para garantizar su existencia y perdurabilidad. $O$ en sentido amplio, incluyendo a cualquier persona o grupo que pueda afectar a las actividades y objetivos de la organización, o que pueda verse afectado por las acciones que la organización lleve a cabo.

Por su parte, James Grunig diferencia entre la responsabilidad pública, que implica atender los compromisos con los públicos directos de la organización, y la responsabilidad social, que supondría atender los compromisos con otros grupos de interés con los que la organización, en principio, no se relacionaría en el desarrollo de las actividades que le son propias. Esta diferenciación entre responsabilidad pública y social, no obstante, no es del todo adecuada a nuestro juicio, pues todos los grupos de interés, tanto aquellos con los que la organización se relaciona directamente, como aquellos otros con los que se pueda relacionar de manera indirecta, constituyen grupos sociales y, por tanto, toda responsabilidad es, en cualquier caso, responsabilidad social.

Sobre esta base, y teniendo en cuenta todo lo expuesto, resulta evidente la enorme importancia adquirida por la educación universitaria en materia de responsabilidad social, en el proceso formativo de los futuros profesionales. Sin embargo, nos preguntamos si estos valores están presentes en la oferta formativa universitaria, a fin de lograr de forma eficaz, que sean aprehendidos, aprendidos y adoptados por los estudiantes que actualmente cursan estudios en nuestras universidades, de manera que en un futuro no lejano puedan aplicarlos en nuestra sociedad.

\subsection{La Ordenación de las enseñanzas universitarias en España}

Para entender el papel de la responsabilidad social corporativa como materia específica en los planes de formación universitaria, es preciso contextualizarlo haciendo referencia al sistema de ordenación de las enseñanzas universitarias. Para ello debemos referirnos al Real Decreto 1393/2007, de 29 de octubre, que regula la ordenación y la estructura de las materias de enseñanzas universitarias en España, adaptando las líneas generales derivadas del Espacio Europeo de Educación Superior y de conformidad con lo previsto en el artículo 37 de la Ley Orgánica de Universidades, 4/2007, de 12 de abril.

Este Real Decreto, en el punto 2 del Artículo 12 de Directrices para el diseño de títulos de Graduado, indica lo siguiente:

Los planes de estudios tendrán 240 créditos, que contendrán toda la formación teórica y práctica que el estudiante deba adquirir: aspectos básicos de la rama de conocimiento, materias obligatorias u optativas, seminarios, prácticas externas, trabajos dirigidos, trabajo de fin de Grado u otras actividades formativas (BOE num.260, de 30 de octubre de 2007: 40040)

Así, las materias o asignaturas en las enseñanzas de Grado pueden definirse de acuerdo con la siguiente tipología:

- Materias correspondientes a la formación básica. Este tipo de asignaturas deberán sumar hasta un total de 60 créditos ECTS.

- Materias obligatorias.

- Materias optativas.

- Prácticas externas (si las hubiere).

- Trabajo Fin de Grado 
A cada materia le corresponde un número determinado de créditos ECTS (sistema europeo de transferencia de créditos). Con la aplicación de este sistema europeo se valorarán las horas que el estudiante debe dedicar al estudio de esa materia. De acuerdo con este criterio, un ECTS representa entre 25 y 30 horas de estudio por parte del alumno, en el que se incluye el tiempo de asistencia a las clases u horas lectivas, las horas de estudio, el tiempo de atención en las tutorías, asistencia a seminarios, realización de trabajos, proyectos o prácticas, además de las horas necesarias para la preparación y realización de exámenes y pruebas de evaluación.

La guía docente de cada asignatura debe indicar con claridad y precisión los siguientes extremos:

- Denominación.

- Objetivos. Competencias que adquiere el estudiante.

- Breve descripción de sus contenidos.

- Actividades formativas con su contenido en créditos ECTS, su metodología de enseñanza-aprendizaje y su relación con las competencias que debe adquirir el estudiante.

- Sistema de evaluación de la adquisición de las competencias y sistema de calificaciones de acuerdo con la legislación vigente.

En relación con las Competencias generales y específicas que los estudiantes deben adquirir durante sus estudios, exigibles para otorgar el título, el Real Decreto 1393/2007, de 29 de octubre, por el que se establece la ordenación de las enseñanzas universitarias oficiales, en su Anexo I, punto 3, indica lo siguiente:

3.1 Las competencias propuestas deben ser evaluables. 3.2 Se garantizarán, como mínimo las siguientes competencias básicas, en el caso del Grado, y aquellas otras que figuren en el Marco Español de Cualificaciones para la Educación Superior, MECES: Que los estudiantes hayan demostrado poseer y comprender conocimientos en un área de estudio que parte de la base de la educación secundaria general, y se suele encontrar a un nivel que, si bien se apoya en libros de texto avanzados, incluye también algunos aspectos que implican conocimientos procedentes de la vanguardia de su campo de estudio; Que los estudiantes sepan aplicar sus conocimientos a su trabajo o vocación de una forma profesional y posean las competencias que suelen demostrarse por medio de la elaboración y defensa de argumentos y la resolución de problemas dentro de su área de estudio; Que los estudiantes tengan la capacidad de reunir e interpretar datos relevantes (normalmente dentro de su área de estudio) para emitir juicios que incluyan una reflexión sobre temas relevantes de índole social, científica o ética; Que los estudiantes puedan transmitir información, ideas, problemas y soluciones a un público tanto especializado como no especializado; Que los estudiantes hayan desarrollado aquellas habilidades de aprendizaje necesarias para emprender estudios posteriores con un alto grado de autonomía (BOE num.260, de 30 de octubre de 2007:40046).

De acuerdo con esta regulación jurídica, todas las materias deberían conducir a una formación en responsabilidad social, pues todas las asignaturas deben propiciar al estudiante la capacidad de reflexionar sobre temas éticos y sociales, además de científicos. Nosotros, no obstante, no nos planteamos estudiar todas las materias que se imparten en todos los grados de la universidad española, sino sólo aquellas que incluyen en su denominación los términos de "responsabilidad social", y sólo en el área de las ciencias sociales, en concreto, en los títulos de grado vinculados al ámbito de la comunicación. 


\section{Objetivos e Hipótesis}

Como ya hemos señalado, nuestra sociedad demanda profesionales cada vez más responsables, con valores y conductas fundamentadas en el respeto y la consideración de los intereses de los diferentes grupos sociales. Y las universidades desempeñan un papel fundamental en el proceso de formar y transmitir esta actitud socialmente responsable.

Necesitamos una sociedad comprometida y responsable en todos los ámbitos. Necesitamos hombres y mujeres, líderes, futuros jefes, directivos, gerentes y gestores que sean conscientes y asuman las consecuencias del su estilo de gobierno, de sus decisiones, sus conductas y su comunicación, y que conozcan y transmitan los valores de la responsabilidad social a la organización a la que pertenecen.

Partiendo del enfoque de gestión de las relaciones con los grupos de interés de Feeman y Reed (1983) y apoyándonos en la teoría de las relaciones publicas excelentes, socialmente responsables (Grunig,1992), como ya hemos mencionado, nos proponernos conocer si en las universidades españolas se están llevando a cabo programas educativos en materia de responsabilidad social corporativa, en sus estudios de grado, en el ámbito de la comunicación, con el fin de formar futuros profesionales suficientemente preparados en esta materia.

Además una vez identificados los programas formativos existentes, profundizaremos en ellos más detalladamente. Se trata de conocer y analizar las características de las diferentes guías docentes de las asignaturas relacionadas con la RSC impartidas en los grados universitarios, de acuerdo con la ordenación de enseñanza superior en España.

Del largo listado de grados oficiales que ofrece la universidad española, serán objeto de este estudio los grados pertenecientes al ámbito de la comunicación: Publicidad y RR.PP, Comunicación Audiovisual, Comunicación, Marketing, Protocolo y Periodismo.

Y sobre esta base, nos planteamos los siguientes objetivos específicos:

- Identificar el grado de presencia de programas de formación en RSC en las universidades españolas.

- Describir el contenido de las guías docentes de las asignaturas de RSC en los grados pertenecientes al ámbito de la comunicación, en función de una serie de indicadores, y compararlos con las de otros títulos de diferentes disciplinas de las ciencias sociales, en la universidad española.

Y formulamos las siguientes hipótesis:

- Los programas de formación en materia de RSC no se han implantado de forma progresiva y efectiva en las universidades españolas, como se plantea en la Estrategia española 2014-2020.

- Las competencias que se adquieren en los diferentes programas formativos sobre RSC difieren entre sí dependiendo del ámbito científico al que pertenece el grado universitario.

\section{Metodología}

La presente investigación constituye una importante aportación en el diagnóstico de las carencias formativas de nuestros estudiantes y futuros profesionales en materia de responsabilidad social. Y paliarlas es ya una necesidad imperiosa. La 
sociedad se muestra cada vez más censora y crítica, y menos permisiva con los comportamientos no responsables, no fundados en principios éticos, no asentados en criterios de sostenibilidad, y no propicios al desarrollo social.

La presente investigación constituye un estudio de campo, de tipo descriptivo y carácter documental, con el objetivo de analizar, inferir y extraer una serie de conclusiones y resultados sobre la formación en materia de RSC llevada a cabo en las universidades españolas.

Con el fin de obtener una mayor información tomaremos el listado total de universidades españolas, que procederemos a segmentar oportunamente. De este modo, analizaremos, por un lado las universidades públicas y, por otro, las universidades privadas.

La investigación está enfocada directamente a analizar los programas formativos en materia de RSC de los grados que se imparten en 2018 en estas universidades españolas.

En total se ha realizado un análisis de 50 universidades de carácter público, y 26 universidades de carácter privado.

Para el examen de las asignaturas de RSC, tomamos como referencia las guías docentes de cada una de ellas en el plan de estudios del correspondiente grado universitario. Y para su análisis hemos adoptado los criterios de ordenación de las enseñanzas universitarias en España, centrándonos, en primer lugar, en las características comunes de cada asignatura (universidad, facultad, grado, modulo, materia, asignatura, carácter y perfil docente), procediendo, posteriormente, al examen de las competencias específicas descritas en cada una.

Nos centramos en las competencias específicas, excluyendo las competencias generales relativas a la titulación, debido a su relación directa con el contenido y conocimiento que se pretende transmitir en cada asignatura en concreto.

Toda esta información permite observar el estado actual en que se encuentran los planes de formación y explorar los diferentes enfoques de las diversas asignaturas.

Para ordenar toda esta información se ha procedido a la estructuración mediante tablas relativas a cada una de las universidades (privadas/publicas), cada uno de sus grados objeto de estudio, y finalmente las asignaturas encontradas.

Previo al estudio de los diferentes documentos, hemos definido una serie de categorías y variables con el fin de organizar la información relevante y útil a la hora de analizar el contenido docente.

La elección de dichas variables o indicadores se ha realizado a partir de la información recabada mediante dos entrevistas en profundidad a expertos, un docente del área de la comunicación, y otro del área de las Ciencias Económicas y finanzas. Y desde el apoyo documental de la investigación llevada a cabo por López Regalado, Perusquía Velasco, Bernal Escoto, Villalón Cañas y Zarate Cornejo (2015).

Los indicadores identificados para segmentar y analizar las competencias de las asignaturas sobre RSC son los siguientes:

- Valores (éticos, de conducta, de liderazgo...).

- Transparencia.

- Buen gobierno corporativo.

- Público interno.

- Público externo. 
- Medio ambiente y Sostenibilidad.

- Proveedores.

- Comunidad.

- Gobierno y Sociedad.

\section{Resultados}

Obtenido el listado total de universidades españolas, se ha procedido a tabular los programas docentes de cada asignatura de los grados, con sus características principales. Se trata de una serie de tablas que contienen información sobre las características recogidas en la ordenación de las enseñanzas españolas. En dichas tablas recogemos todas las asignaturas sobre RSC impartidas en la totalidad de las universidades españolas.

Además se incorpora en ellas la información relativa a los diferentes perfiles docentes del profesorado que imparte esas asignaturas.

Los grados universitarios en los que comprobamos presencia de formación en RSC son: en Administración, Dirección y creación de empresas; en Finanzas, Contabilidad, Banca, Seguros, Economía, Marketing, Derecho, Relaciones laborales, Relaciones internacionales, Publicidad y Relaciones públicas, Comunicación audiovisual, Protocolo y Periodismo.

Una vez llevado a cabo el estudio documental de cada guía docente, y habiendo identificado todas las asignaturas existentes, procedemos a realizar un análisis que nos proporcione una imagen actual de la formación en RSC en las universidades españolas.

\subsection{Análisis de las universidades que imparten formación de RSC en sus diferentes grados}

En primer lugar debemos destacar el número de universidades en las que hemos encontrado esta formación específica en RSC.

En el caso de las organizaciones universitarias públicas observamos como de un total de 50 universidades analizadas, hoy se ofrecen asignaturas en materia de RSC en 20 de ellas.

Se trata de un resultado no muy elevado. El $40 \%$ del total de las universidades públicas ofrece esta formación, llegando a este porcentaje desde que en 2014 se comenzase con la estrategia formativa como una de las principales líneas de actuación en cuanto a responsabilidad social corporativa. 
Cuadro 1. Universidades públicas que incorporan asignaturas de RSC en sus grados.

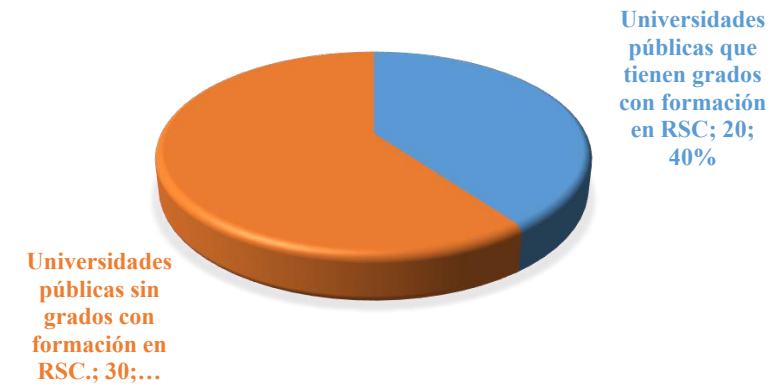

Fuente: elaboración propia

En las organizaciones universitarias privadas detectamos algunas diferencias. En este caso, se ofrece formación en materia de RSC en un 54\% de las universidades. Aunque no es un resultado prominente, si es destacable que este tipo de formación esté presente en más de la mitad de estos centros. Un 14\% más que en las universidades públicas.

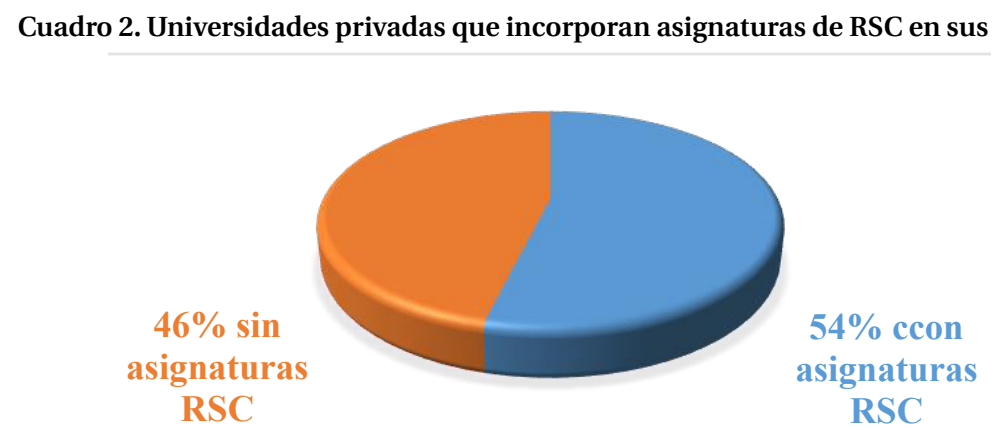

Fuente: elaboración propia

4.2. Análisis de las asignaturas de RSC impartidas en las universidades españolas, atendiendo al tipo de materia

En el apartado anterior observábamos el número de universidades, públicas y privadas, que ofrecen formación de RSC es sus grados. Ahora ponemos el foco de atención en las asignaturas. Se trata de detectar y segmentar las asignaturas de las universidades españolas, según el tipo de materia establecido en la Ordenación de enseñanza española (formación básica, obligatoria, optativa), y el tipo de institución a la que pertenecen. 
Así, refiriéndonos en primer lugar a las universidades públicas, encontramos un total de 24 asignaturas sobre RSC impartidas en los grados analizados, destacando en ellas el elevado número de materias con carácter optativo. El 69\%, de las asignaturas sobre RSC son optativas. El 17\% tienen carácter obligatorio. Y el 4\% son asignaturas de formación básica.

Estos datos resultan comprensibles debido al reciente desarrollo de este tipo de formación en las universidades españolas y a la posibilidad de cada universidad de definir el plan de estudios y el carácter de cada asignatura, adaptándolo a las necesidades del grado en que se imparten.

Con estos resultados nos planteamos la duda de si los contenidos en materia de RSC, debido precisamente a su demanda por parte de la sociedad en el mundo empresarial, económico, político, y la importancia que en los últimos años está cobrando, debería tener mayoritariamente un carácter obligatorio o de formación básica, en lugar de ofrecerse como materia optativa en cualquiera de los grados analizados. Pues, planteándose como asignatura obligatoria, se garantizaría que todos los egresados hubieran adquirido formación específica en materia de responsabilidad social corporativa.

Cuadro 3. Número de asignaturas de RSC impartidas en grados de las universidades públicas españolas. Segmentación según el tipo de materia.

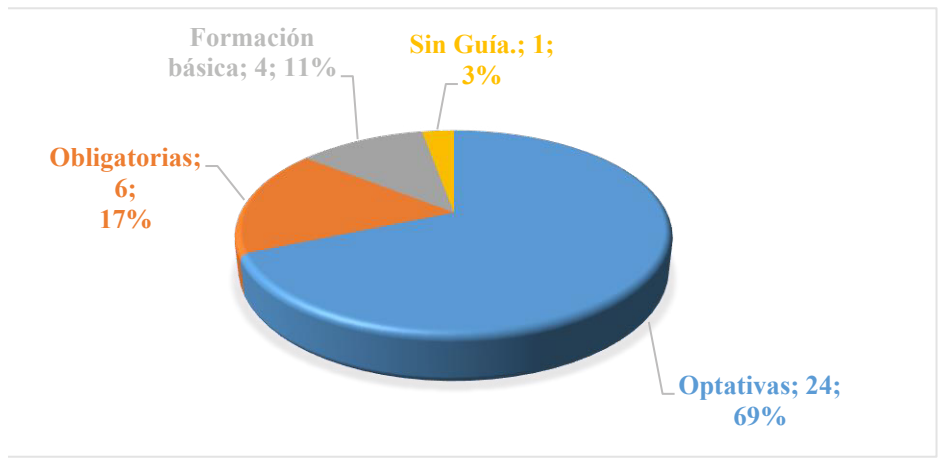

Fuente: elaboración propia

En el ámbito de las universidades privadas, observamos, sin embargo, algunas diferencias significativas, obteniendo resultados opuestos a los de las universidades públicas. En las universidades privadas, el $67 \%$ del total de las asignaturas de RSC de los grados analizados corresponde a materias obligatorias, seguido de un $27 \%$ de asignaturas adscritas a la formación básica y, en última instancia, tan solo el $6 \%$ de estas materias posee carácter de optatividad.

Así, podemos observar claramente que la tendencia en cuanto a la naturaleza de las materias, en el caso de los grados pertenecientes a organizaciones universitarias privadas, correlaciona de manera inversa con las de las universidades públicas, primando sobre todo la obligatoriedad de la formación en RSC. 
Cuadro 4. Número de asignaturas de RSC impartidas en grados de las universidades privadas españolas. Segmentación según el tipo de materia.

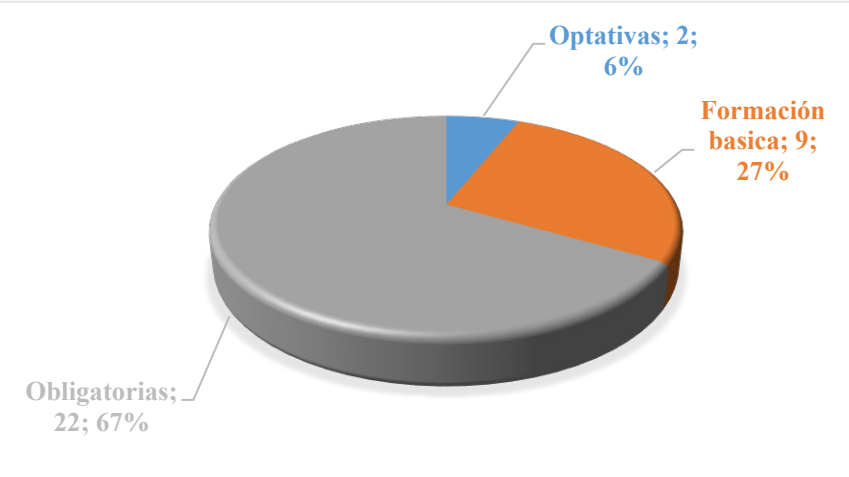

Fuente: elaboración propia

\subsection{Análisis de los diferentes grados universitarios con asignaturas formativas en RSC}

Fijamos nuestra atención, ahora, en los grados universitarios que comprenden asignaturas sobre RSC.

Debemos saber que en el listado completo de los grados analizados encontramos algunos cuya denominación puede ser diferente, aun refiriéndose al mismo tipo de estudios universitarios. Este es el caso, por ejemplo, de los grados en Administración y dirección de empresas o Dirección y creación de empresas.

Para tener en cuenta esta circunstancia en nuestro análisis, hemos agrupado los grados universitarios en bloques, de manera que todos los grados de una misma disciplina han sido agregados en un único bloque temático.

En total, el número de grados universitarios con formación en RSC asciende a 35 en el caso de las universidades públicas, y a 33 en el caso de las universidades privadas.

\subsubsection{La RSC en los grados de las universidades públicas}

De los resultados obtenidos en relación con los grados analizados en las universidades públicas españolas, señalamos los siguientes datos.

Destacan, en primer lugar, los grados agrupados dentro de la disciplina de Administración y dirección de empresas, que comprenden un 37\% del total de grados universitarios pertenecientes al ámbito público con formación en RSC. En segundo término, encontramos los grados enmarcados en el ámbito de Finanzas, Contabilidad, Banca y Seguros, que representan el $20 \%$ del total de los grados universitarios públicos. Tan sólo el 10\% de los grados que imparten formación sobre RSC pertenecen al ámbito de la Economía, Publicidad y RR.PP. y Relaciones laborales, respectivamente. 
Por último, y de forma mucho menos relevante, encontramos formación sobre RSC en los grados de Marketing (5\%), de Gestión y Administración pública (3\%) y Protocolo (3\%). Y, finalmente, hallamos un solo grado en Comunicación Audiovisual $(2 \%)$ que imparte este tipo de formación como materia específica.

Cuadro 5. Número de grados de universidades públicas que contienen formación en materia de RSC.

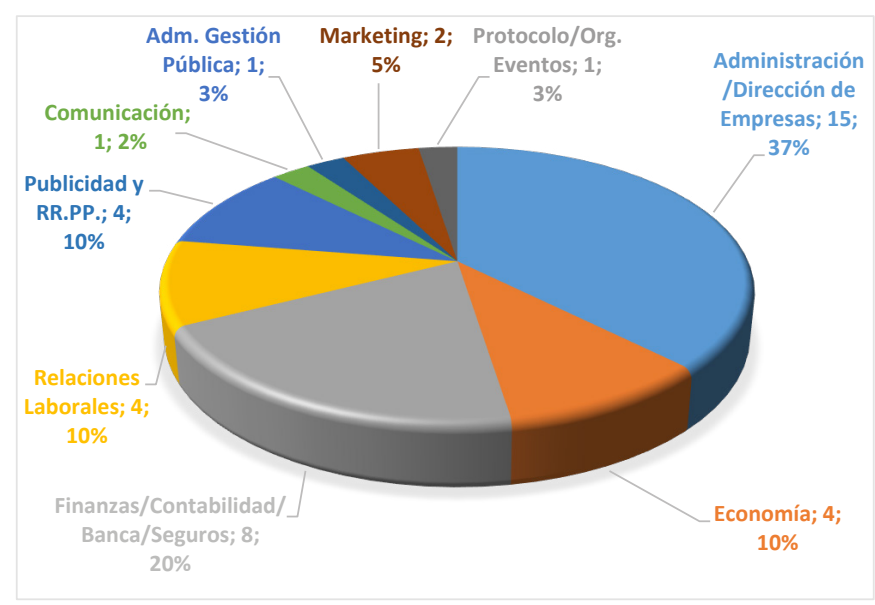

Fuente: elaboración propia

Como vemos, cerca del 70\% de las asignaturas de RSC en las universidades públicas se imparten en los grados de Dirección de empresas, Economía y Finanzas.

\subsubsection{La RSC en los grados de las universidades privadas}

En relación con los grados pertenecientes a universidades privadas españolas, obtenemos los siguientes datos.

En primer lugar, un 33\% del total de los grados, que ofrecen formación de RSC en las universidades privadas, pertenece a la disciplina de Administración y dirección de empresas.

En segundo lugar, destacan los grados en disciplinas de Marketing y Comunicación, representando el 14\%, respectivamente, de los grados con formación específica en RSC.

Los grados en Publicidad y RR.PP. constituyen el $12 \%$ del total de grados con formación en RSC, seguidos de los grados de Periodismo (9\%), Economía (7\%), Relaciones internacionales (5\%), CC Políticas, Derecho y Protocolo, con un $2 \%$ respectivamente. 
Cuadro 6. Número de grados de universidades privadas que contienen formación en materia de RSC.

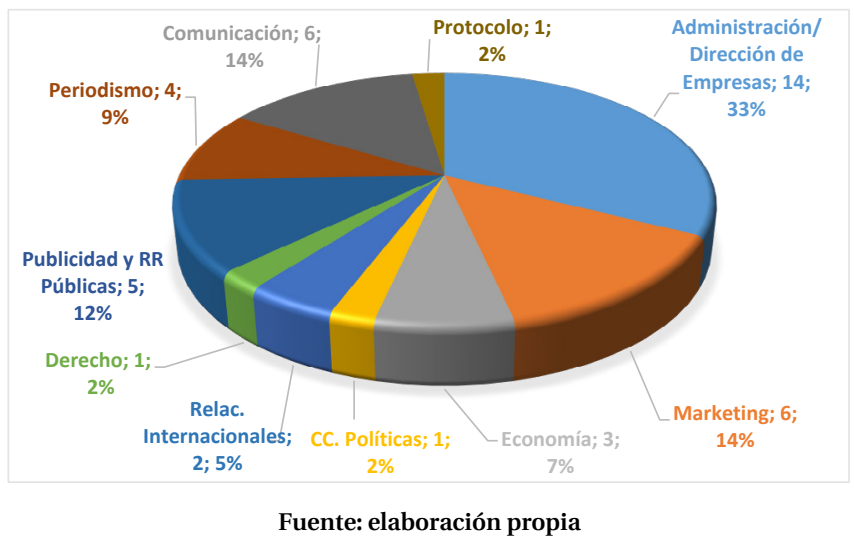

Tanto en las universidades privadas, como en las universidades públicas, el ámbito disciplinar en el cual se presenta el mayor catálogo de formación en RSC es el de Administración y dirección de empresas, con una presencia de algo más del $30 \%$ en ambos grupos de universidades.

Cabe destacar las diferencias detectadas en los demás grados universitarios, entre las universidades públicas y las privadas. Recordamos que, en las universidades públicas, el siguiente porcentaje más alto lo encontramos en el ámbito de las Finanzas, Contabilidad, Banca y Seguros, con un 20\%. Mientras que en el caso de las universidades privadas, son las áreas de Marketing y comunicación las que destacan en segundo lugar, con un porcentaje total del $28 \%$.

De cualquier modo, si ponemos nuestra atención en los grados pertenecientes al ámbito específico de la comunicación, observamos, de carácter general, la existencia de un sorprendentemente reducido porcentaje de asignaturas concretas dedicadas a la formación en materia de RSC, frente a los grados pertenecientes al ámbito de la empresa, las finanzas y la economía.

Destaca, también, una mayor presencia de la formación de RSC en los grados pertenecientes al ámbito de la comunicación, en el caso de las universidades privadas, con 15 grados, frente a las universidades públicas, en las que sólo está presente en 6 grados relacionados directamente con la comunicación, la publicidad y las relaciones públicas, el protocolo y la organización de eventos.

\subsection{Resultados de orientación de las competencias específicas adquiridas en las asignaturas de RSC}

En este apartado presentamos los resultados obtenidos del análisis de las competencias específicas referidas en las guías docentes de cada una de las asignaturas de los diferentes grados analizados.

A continuación, en las siguientes tablas, se indica el número de asignaturas encontradas por cada grado y ámbito de aplicación. 
Cuadro 7. Número de asignaturas de RSC identificadas y analizadas en universidades públicas.

\begin{tabular}{|c|c|c|}
\hline \multicolumn{2}{|c|}{ Universidades públicas } \\
\hline Grados & Ámbito & Número de asignaturas \\
\hline Administración/Dirección de Empresas & Economía / Empresa & 13 \\
\hline Finanzas/Contabilidad/Banca/Seguros & Economía / Empresa & 8 \\
\hline Economía & Economía / Empresa & 4 \\
\hline Relaciones laborales & Economía / Empresa & 4 \\
\hline Publicidad y RR.PP. & Comunicación & 4 \\
\hline Marketing & Comunicación & 2 \\
\hline Gestión y Administración pública & Administración & 1 \\
\hline Comunicación audiovisual & Comunicación & 1 \\
\hline Protocolo, Organización de Eventos y Comunicación Corporativa & Comunicación & 1 \\
\hline
\end{tabular}

Fuente: elaboración propia

Cuadro 8. Número de asignaturas sobre RSC identificadas y analizadas en universidades privadas.

\begin{tabular}{|c|c|c|}
\hline \multicolumn{2}{|c|}{ Universidades privadas } \\
\hline Grados & Ámbito & Número de asignaturas \\
\hline Administración/Dirección de Empresas. & Economía / Empresa. & 6 \\
\hline Marketing. & Comunicación. & 5 \\
\hline Publicidad y RR.PP. & Comunicación. & 4 \\
\hline Comunicación. & Comunicación. & 3 \\
\hline Periodismo. & Comunicación. & 2 \\
\hline Economía. & Economía / Empresa. & 1 \\
\hline Relaciones Internacionales. & Economía / Empresa. & 1 \\
\hline Protocolo. & Comunicación. & 1 \\
\hline Ciencias políticas. & Jurídico. & \\
\hline Derecho. & Jurídico. & \\
\hline
\end{tabular}

Fuente: elaboración propia

Esta distribución de las asignaturas de RSC, en las universidades públicas y privadas, incide, como ya hemos visto anteriormente, en un claro predominio del enfoque empresarial y financiero de la RSC en las universidades públicas frente a las privadas. 
4.4.1. Orientación de las competencias específicas adquiridas en las diferentes asignaturas de RSC pertenecientes a universidades públicas

Veamos las diferencias de las competencias descritas en las guías docentes de los títulos de grado en las universidades públicas, en función del ámbito científico.

- Administración/Dirección de empresas.

Partiendo de un total de 13 asignaturas relativas a la RSC impartidas en esta disciplina, obtenemos los siguientes resultados reflejados en el Cuadro 9.

En este caso, comprobamos como entre los indicadores más representados en la descripción de las competencias, destacan los valores, presentes en un total de 7 asignaturas; gobierno y sociedad, presentes en 5 de ellas; y en tercer lugar, medio ambiente y sostenibilidad, presente en 3 asignaturas.

Parece lógico comprobar como los indicadores de RSC de las guías docentes en el ámbito de las ciencias empresariales están claramente relacionados mayoritariamente con las tareas propias de los puestos de gestión y dirección en las organizaciones.

Sin embargo, resulta destacable la escasa presencia de los indicadores de transparencia, cuando, sabemos, este indicador constituye uno de los más importantes y demandados en este tipo de disciplinas.

Cuadro 9. Orientación de las competencias específicas en las asignaturas de RSC impartidas en grados de Administración/Dirección de empresas (universidades públicas).

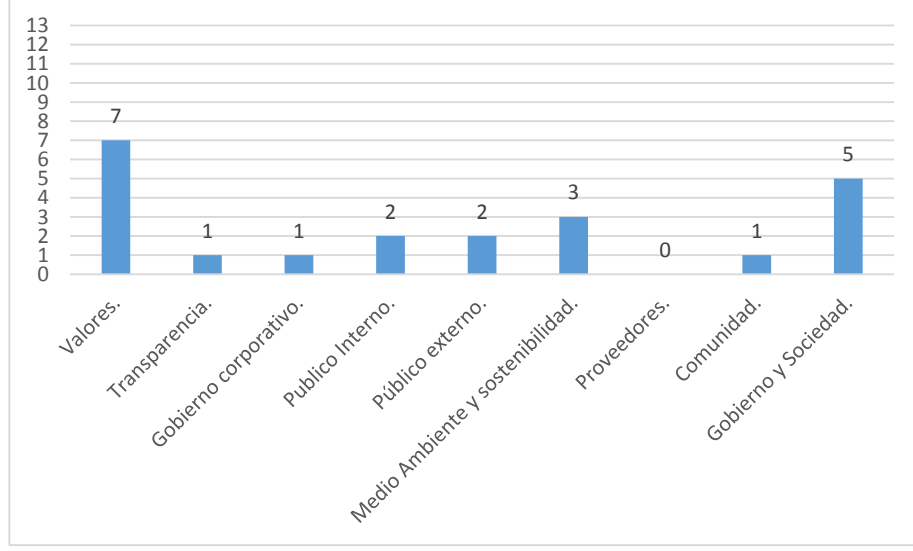

Fuente: elaboración propia 
- Finanzas/Contabilidad/Banca/Seguros.

En esta disciplina encontramos un total de 8 asignaturas en las que cabe destacar tres indicadores.

En primer lugar, la transparencia, presente en un total de 6 de las asignaturas; en segundo lugar, los valores, en 5 de ellas; y en tercer lugar, el buen gobierno corporativo, presente en 3 asignaturas.

Vemos, en este caso, como aparece con mayor frecuencia el indicador transparencia. Posiblemente, como consecuencia del tipo de disciplina, pues ésta se orienta principalmente hacia la gestión de cuentas y finanzas y, en definitiva, implica gestiones que exigen obrar de buena fe y con la mayor transparencia posible.

Cuadro 10. Orientación de las competencias específicas en las asignaturas de RSC impartidas en grados de Finanzas/Contabilidad/Banca/Seguros (universidades públicas).

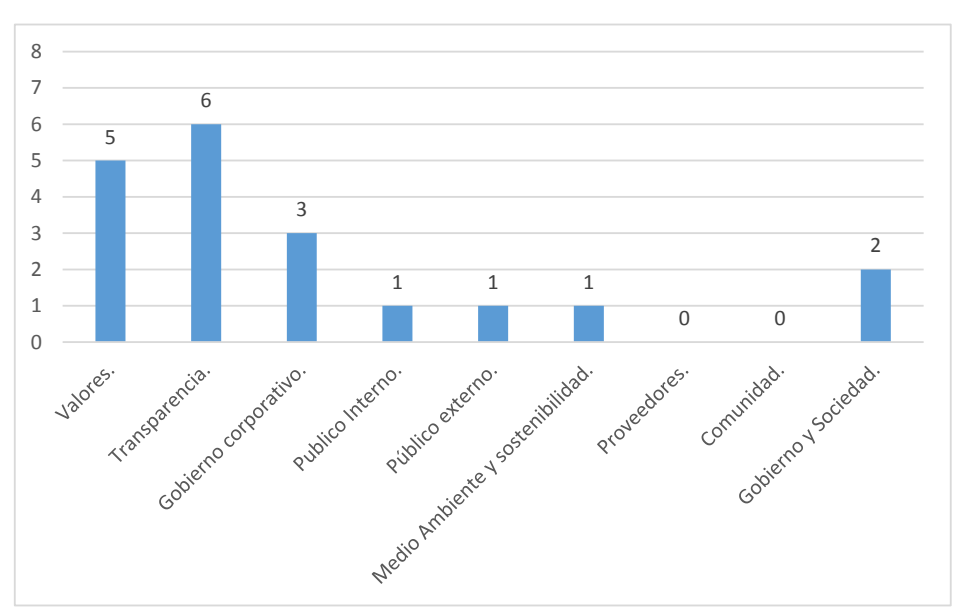

Fuente: elaboración propia

- Economía.

En los grados de economía la presencia de asignaturas sobre RSC es baja, con un total de 4 asignaturas.

En dos de estas cuatro asignaturas aparece como indicador el medio ambiente y la sostenibilidad, seguido de un reparto equitativo en competencias que trasmiten valores, buen gobierno corporativo, gestión con los públicos y gobierno y sociedad. 
Cuadro 11. Orientación de las competencias específicas en las asignaturas de RSC impartidas en grados de Economía (universidades públicas).

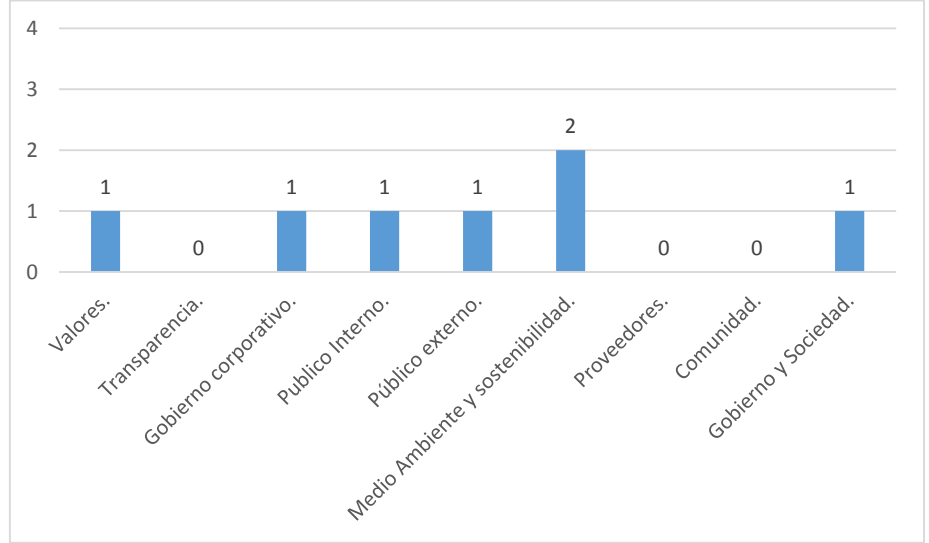

Fuente: elaboración propia

- Relaciones laborales.

En estos grados encontramos un total de cuatro asignaturas, cuyas competencias a destacar se relacionan, fundamentalmente, con la adquisición de valores y competencias en el buen gobierno corporativo.

Cuadro 12. Orientación de las competencias específicas en las asignaturas de RSC impartidas en grados de Relaciones Laborales (universidades públicas).

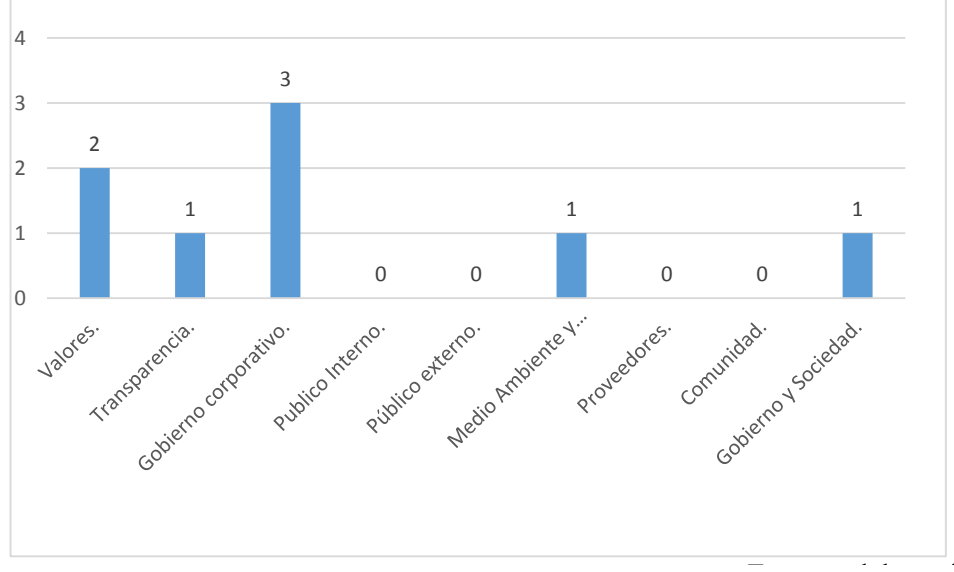

Fuente: elaboración propia 
- Gestión y Administración pública.

$\mathrm{Al}$ igual que en el caso anterior, solo existe una asignatura en esta disciplina, que comprende competencias sobre Valores y gobierno/sociedad.

- Publicidad y RR.PP.

En los estudios de Publicidad y RR.PP. con un total de cuatro asignaturas impartidas, observamos que sus competencias van de la mano con la transmisión de valores, buen gobierno corporativo y temas relacionados con gobierno y sociedad.

Cuadro 13. Orientación de las competencias específicas en las asignaturas de RSC impartidas en grados de Publicidad y RR.PP. (universidades públicas).

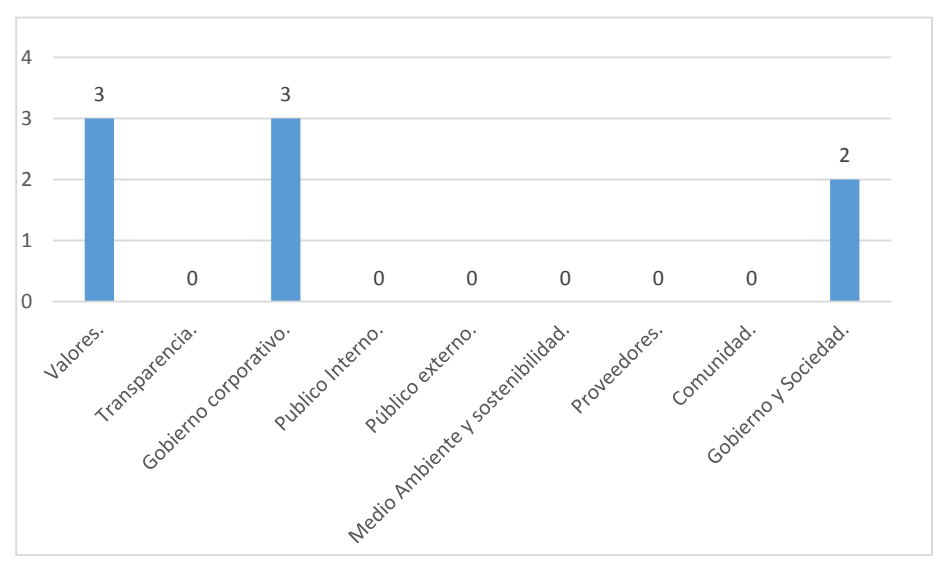

Fuente: elaboración propia

- Comunicación audiovisual.

Encontramos una sola asignatura y un único grado que la imparte, destacando en sus competencias la transmisión de valores y la gestión de sus funciones atendiendo al gobierno y sociedad.

- Marketing.

Sólo descubrimos dos asignaturas con competencias ligadas al buen gobierno corporativo y la gestión de sus funciones frente a la Sociedad. 
Cuadro 14. Orientación de las competencias específicas en las asignaturas de RSC impartidas en grados de Marketing (universidades públicas).

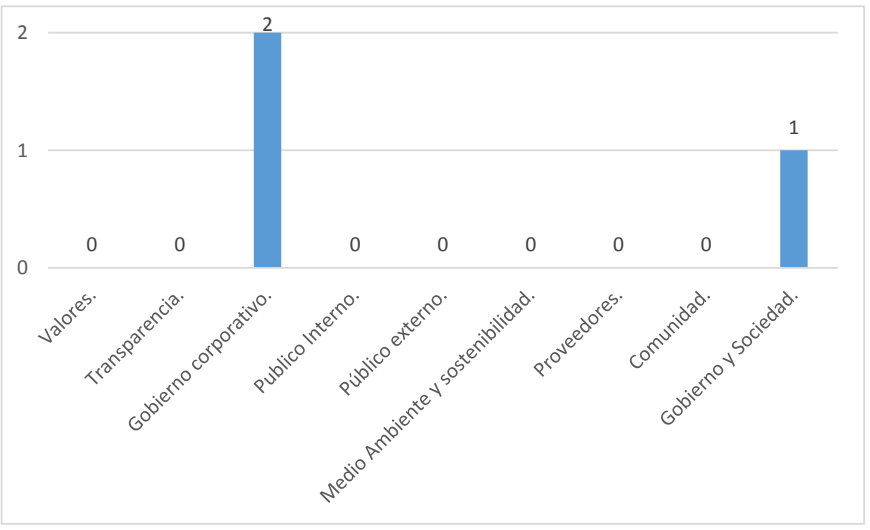

Fuente: elaboración propia

- Protocolo, Organización de Eventos y Comunicación Corporativa.

Sólo hallamos una asignatura de RSC ligada a la transmisión de conocimientos relacionados con valores, gobierno y sociedad.

\subsubsection{Orientación de las competencias específicas adquiridas en los grados pertenecientes a universidades privadas}

Veamos las diferencias de las competencias descritas en las guías docentes de los títulos de grado en las universidades privadas, en función del ámbito científico.

- Administración/Dirección de Empresas.

Obteniendo el mismo número de asignaturas, 13, que en los mismos grados de las universidades públicas, observamos como en los grados de Administración y Dirección de empresas en universidades privadas, las competencias se orientan hacia los valores (en 9 asignaturas) y buen gobierno corporativo (10 asignaturas).

Básicamente se enfocan en los valores éticos, comportamientos, principios y normas que regulan el diseño, integración y funcionamiento de los órganos de gobierno de la empresa.

En el caso de las universidades públicas, los valores también toman una fuerte presencia, pero no es así en el caso del buen gobierno corporativo. 
Cuadro 15. Orientación de las competencias específicas en las asignaturas de RSC impartidas en grados de Administración/ Dirección de Empresas (universidades privadas).

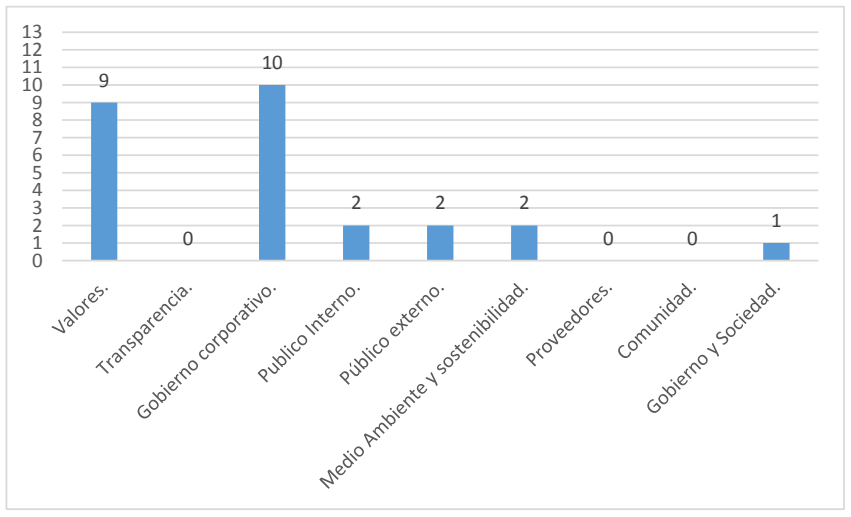

Fuente: elaboración propia

- Marketing.

En cuanto a las competencias específicas de los grados de Marketing en universidades privadas, del total de asignaturas (6), destacan el buen gobierno corporativo (presente en 6 asignaturas), los valores (5 asignaturas) y Medio Ambiente y Sostenibilidad (3 asignaturas).

Vemos que se repiten los dos primeros indicadores respecto de los grados de Administración/Dirección de Empresas y además toma protagonismo el Medio Ambiente y la Sostenibilidad.

Cuadro 16. Orientación de las competencias específicas en las asignaturas de RSC impartidas en grados de Marketing (universidades privadas).

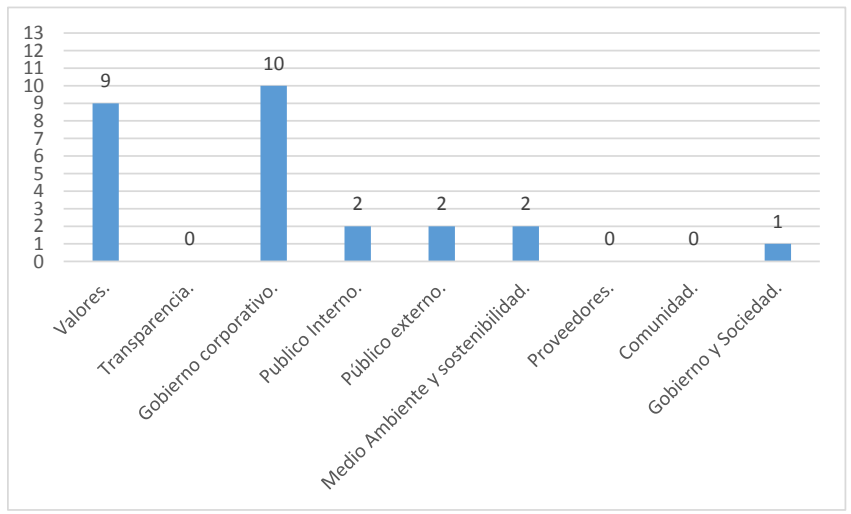

Fuente: elaboración propia 
- Economía.

La presencia de los grados de economía con materias de RSC es baja. Tan sólo se especifican 3. En este caso encontramos la presencia de buen gobierno corporativo en primer lugar, seguido de valores, Medio Ambiente, y gobierno y sociedad. Indicadores que están presentes en los mismos grados de las organizaciones universitarias públicas.

Cuadro 17. Orientación de las competencias específicas en las asignaturas de RSC impartidas en grados de Economía (universidades privadas).

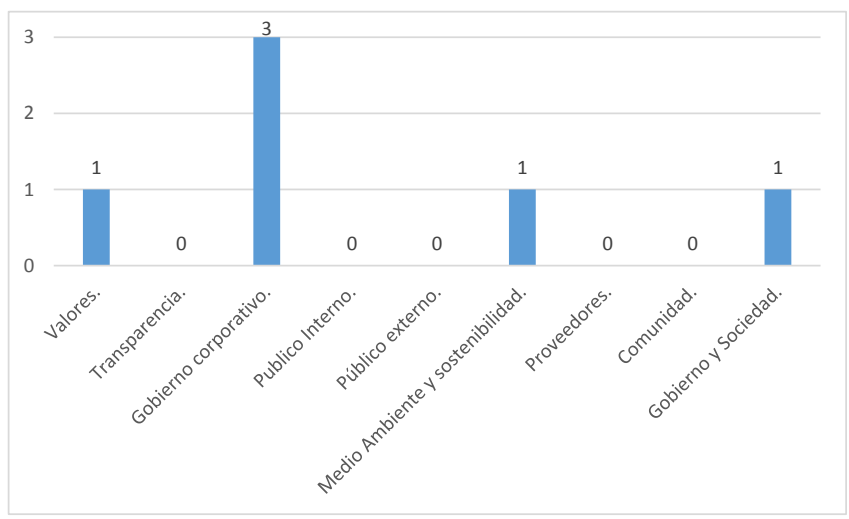

Fuente: elaboración propia

- Ciencias políticas.

Única asignatura impartida con competencias de valores y buen gobierno corporativo.

- Relaciones Internacionales.

Dos asignaturas impartidas con competencias de valores, buen gobierno corporativo y sociedad.

- Derecho.

Única asignatura impartida con competencias de valores y buen gobierno corporativo.

- Publicidad y Relaciones Públicas.

Encontramos 5 asignaturas sobre RSC, las cuales destacan por hacer hincapié en valores éticos, la buena gestión frente a la Sociedad, y mínimamente en el buen Gobierno Corporativo. 
Cuadro 18. Orientación de las competencias específicas en las asignaturas de RSC impartidas en grados de Publicidad y RR.PP. (universidades privadas).

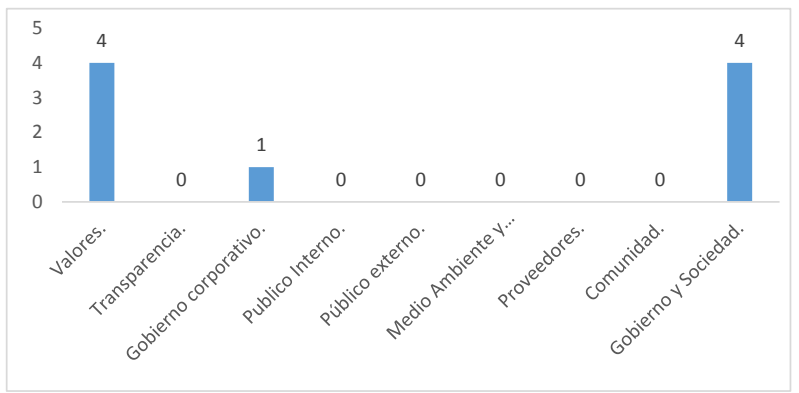

Fuente: elaboración propia.

- Periodismo.

Las 4 asignaturas impartidas en grados de Periodismo, destacan por una formación ligada a valores, buen gobierno corporativo, y gestión frente a la sociedad.

Cuadro 19. Orientación de las competencias específicas en las asignaturas de RSC impartidas en grados de Periodismo (universidades privadas).

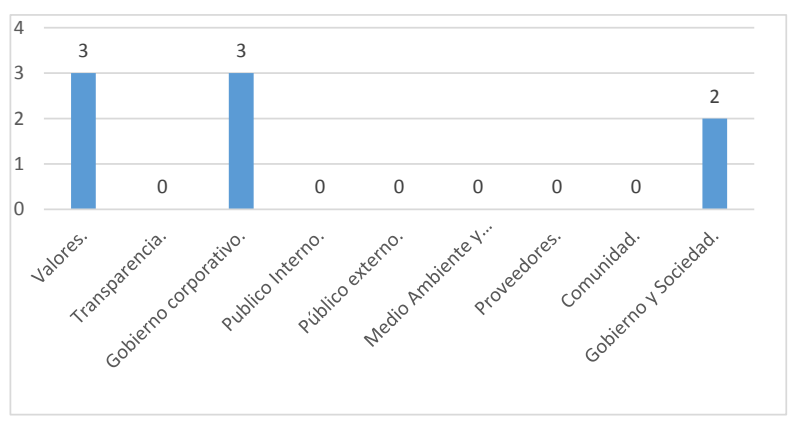

Fuente: elaboración propia.

- Comunicación.

En cuanto a los grados de Comunicación, las asignaturas de RSC (5 asignaturas), transmiten competencias ligadas a valores, gobierno corporativo, y gobierno y sociedad. 
Cuadro 20. Orientación de las competencias específicas en las asignaturas de RSC impartidas en grados de Comunicación (universidades privadas).

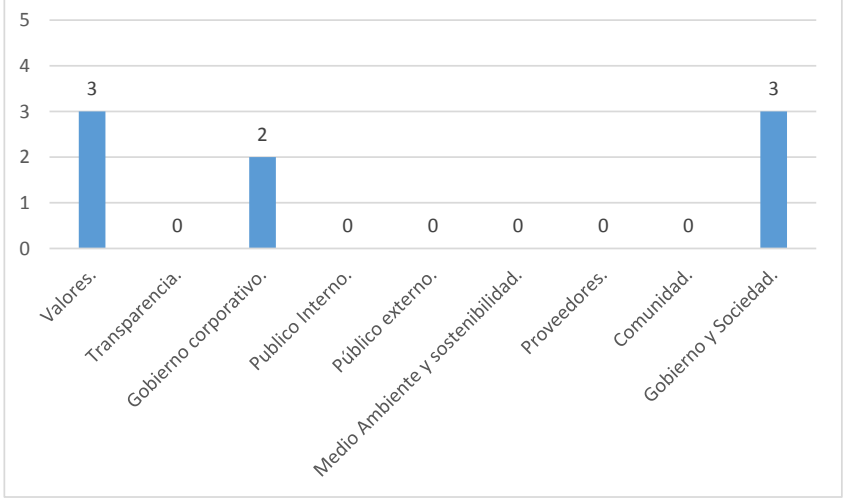

Fuente: elaboración propia

- Protocolo.

En este caso encontramos una sola asignatura en la cual se transmiten, ante todo, valores y técnicas para un buen gobierno corporativo.

\subsection{Los perfiles docentes del profesorado de las asignaturas sobre RSC}

Veamos los diferentes perfiles docentes en el las materias del ámbito de la comunicación, por un lado, y en el de las otras diversas disciplinas, por otro.

\subsubsection{Los perfiles docentes del profesorado de las asignaturas sobre RSC en el ámbito de la comunicación}

En este apartado hemos procedido a filtrar y tabular los diferentes perfiles de los docentes que imparten cada una de las asignaturas de RSC en los diferentes grados.

En el cuadro 21, exponemos el listado de los perfiles docentes de quienes imparten asignaturas de RSC en los grados del ámbito de la comunicación. Y observamos como los perfiles más frecuentes corresponden a docentes con estudios relacionados con la Comunicación, Ciencias de la Información, Filosofía, Derecho, Humanidades y Sociología. 
Cuadro 21. Perfil docente en los diferentes grados del ámbito de la comunicación.

\begin{tabular}{|c|c|c|c|c|}
\hline $\begin{array}{l}\text { Grado en Publicidad y } \\
\text { Relaciones públicas }\end{array}$ & Grado en Marketing & $\begin{array}{c}\text { Grado en } \\
\text { Comunicación }\end{array}$ & Grado en Protocolo & Grado en Periodismo \\
\hline $\begin{array}{l}\text { Ciencias de la } \\
\text { Información } \\
(2)\end{array}$ & Economía (3) & Comunicación (3) & Derecho (1) & Filosofía (1) \\
\hline Filosofía (2) & Sociología (1) & Derecho (1) & & Humanidades (1) \\
\hline Comunicación (4) & Psicología (1) & Máster en RSC (1) & & Máster en RSC (1) \\
\hline Derecho (1) & Derecho (1) & $\begin{array}{c}\text { Ciencias de la } \\
\text { información (1) }\end{array}$ & & $\begin{array}{l}\text { Ciencias de la } \\
\text { información (1) }\end{array}$ \\
\hline Sociología (3) & $\begin{array}{c}\text { Ciencias de la } \\
\text { Información (2) }\end{array}$ & & & \\
\hline \multirow[t]{3}{*}{ Humanidades (1) } & Humanidades (2) & & & \\
\hline & Filosofía (2) & & & \\
\hline & C.C. Empresariales (1) & & & \\
\hline
\end{tabular}

Cuadro 22. Nube de los perfiles docentes en los diferentes grados del ámbito de la comunicación.

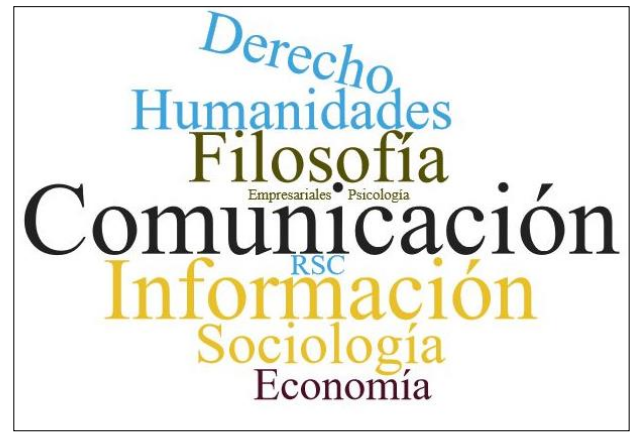

Fuente: elaboración propia

4.5.2. Los perfiles docentes del profesorado de las asignaturas sobre RSC en el resto de grados analizados.

Podemos destacar entre los perfiles docentes del resto de disciplinas, los relacionados con estudios de Economía, Ciencias Empresariales, Filosofía, Derecho y Sociología. 
Cuadro 23. Perfil docente de las materias de RSC en otras titulaciones

\begin{tabular}{|c|c|c|c|c|c|c|}
\hline $\begin{array}{l}\text { Administración/ } \\
\text { Dirección de } \\
\text { Empresas }\end{array}$ & Economía. & $\begin{array}{l}\text { Relaciones } \\
\text { Laborales. }\end{array}$ & $\begin{array}{c}\text { Finanzas/ } \\
\text { Contabilidad/ } \\
\text { Banca/Seguros }\end{array}$ & $\begin{array}{c}\text { Gestión } \\
\text { Adm. Pública. }\end{array}$ & Derecho. & $\begin{array}{c}\text { Relaciones } \\
\text { internacionales }\end{array}$ \\
\hline Sociología (1) & Sociología (1) & Economía (3) & Economía (4) & Derecho (1) & $\begin{array}{c}\text { Ciencias } \\
\text { Empresariales (1) }\end{array}$ & $\begin{array}{l}\text { Ciencias de la } \\
\text { Información (1) }\end{array}$ \\
\hline Economía (8) & $\begin{array}{c}\text { Ciencias Políticas } \\
\text { (1) }\end{array}$ & Sociología (1) & $\begin{array}{c}\text { Ciencias Políticas } \\
(1)\end{array}$ & & & $\begin{array}{l}\text { Ciencias de la } \\
\text { Educación (1) }\end{array}$ \\
\hline $\begin{array}{c}\text { Ciencias } \\
\text { Empresariales (7) }\end{array}$ & Derecho (1) & & Derecho (1) & & & \\
\hline Filosofía (4) & Filosofía (1) & & & & & \\
\hline $\begin{array}{c}\text { Experto en RSC } \\
(1)\end{array}$ & Economía (5) & & & & & \\
\hline $\begin{array}{l}\text { Humanidades } \\
\text { (1) }\end{array}$ & & & & & & \\
\hline Teología (1) & & & & & & \\
\hline
\end{tabular}

Fuente: elaboración propia

Cuadro 24. Nube de los perfiles docentes de las materias de RSC en otras titulaciones.

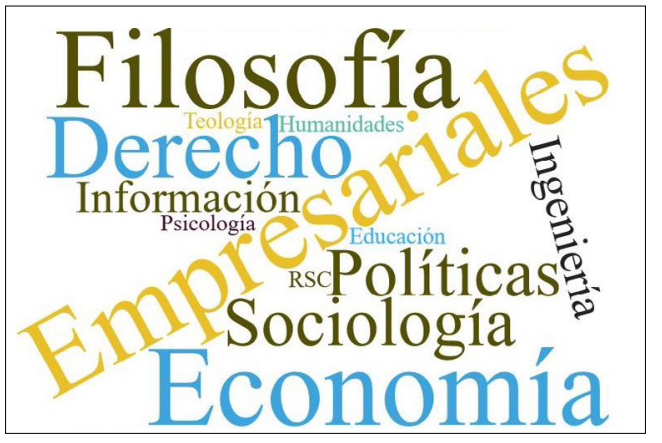

Fuente: elaboración propia 


\section{Conclusiones}

Del análisis de los resultados, extraemos las siguientes conclusiones:

- Eficacia limitada de la implantación de asignaturas de RSC en las universidades españolas.

En nuestros días, en España, encontramos asignaturas específicas en materia de RSC en el 40\% de las universidades públicas españolas y en el $54 \%$ de las universidades privadas.

Podemos concluir, por tanto, que la responsabilidad social corporativa sigue siendo, pese a su progresiva implantación, una materia pendiente para muchas universidades. Si bien, como vemos, las universidades privadas van claramente por delante en este camino de la Estrategia Española de Responsabilidad Social de las Empresas 2014-2020.

- El tipo de materia de las asignaturas impartidas sobre RSC corresponde mayoritariamente a asignaturas optativas en las universidades públicas y a asignaturas obligatorias en las universidades privadas.

El 69\% de las asignaturas sobre RSC impartidas en las universidades públicas tienen carácter optativo. Sin embargo, en las universidades privadas, sólo el $6 \%$ son optativas, mientras que el $67 \%$ de estas asignaturas tienen carácter obligatorio y el $27 \%$ corresponde a asignaturas básicas.

Esta conclusión nos lleva a formular una nueva hipótesis que ahora consideramos plausible y trataremos de verificar en próximas investigaciones. Esto es, el hecho de que en las universidades privadas las asignaturas sobre RSC tengan fundamentalmente carácter obligatorio o básico se debe a la vinculación de algunas de estas universidades privadas con asociaciones o congregaciones católicas con la misión de lograr el bien común de toda la sociedad, lo que las lleva a considerar necesaria la formación específica en relación con la RSC y los comportamientos éticos y responsables. Recordemos, que fue una universidad privada la que incorpora por primera vez, en 1993, la RSC como asignatura curricular en la universidad española.

- Diferente presencia de la RSC en los grados del ámbito de la comunicación.

La conclusión en cuanto a la presencia de formación de RSC en el ámbito de la comunicación es un tanto diferente, dependiendo del tipo de institución.

En las universidades públicas, los grados de Publicidad y Relaciones Públicas, Marketing, Comunicación, Protocolo y Periodismo, con asignaturas de RSC, representan el $20 \%$ del total de grados que incluyen en sus planes de estudios este tipo de materias. Vemos, por tanto, que estos grados del ámbito de la comunicación adquieren menor peso en la formación de la RSC que otros grados correspondientes a otras disciplinas analizadas en este tipo de universidades.

Sin embargo, en las universidades privadas el resultado es el contrario. En estas universidades el $51 \%$ de los grados que ofrecen formación específica en RSC corresponden al ámbito de la comunicación.

- En relación con las competencias específicas de las asignaturas sobre RSC dentro del ámbito de la comunicación, concluimos lo siguiente:

Una vez analizadas las competencias descritas en las guías docentes de las 8 asignaturas sobre RSC impartidas en las universidades públicas, y de las 21 asignaturas identificadas en la oferta de las universidades privadas, comprobamos la coincidencia de que, en ambas, las competencias se orientan principalmente a la transmisión de valores, al desarrollo de 
conductas responsables en el ámbito profesional de las organizaciones en relación con la sociedad y, en las universidades públicas, el buen gobierno corporativo.

- Respecto de las competencias específicas de las asignaturas sobre RSC en los grados del ámbito de las Ciencias empresariales, financieras y económicas, concluimos que, tanto en las universidades públicas como en las privadas, en estas asignaturas se desarrollan fundamentalmente contenidos relacionados con el buen gobierno corporativo y, en menor medida, con otros aceleradores de la RSC como la transparencia en la gestión, la conservación del medio ambiente y la sostenibilidad o los valores.

Y en los grados de Marketing, las asignaturas de RSC se concretan casi exclusivamente en los indicadores del buen gobierno corporativo y en la relación de la gestión empresarial y la sociedad.

- Los perfiles docentes de los profesores de las asignaturas de RSC en el ámbito de la comunicación se diferencian claramente de los correspondientes al profesorado de este tipo de asignaturas del ámbito de las ciencias económicas y empresariales. El perfil docente más destacado en las asignaturas de RSC en el ámbito de la comunicación aparece vinculado a los estudios de Comunicación, Ciencias de la Información, Filosofía, Humanidades o Derecho. Sin embargo, fuera del ámbito de la comunicación, los perfiles docentes más notorios se relacionan principalmente con las Ciencias empresariales, la Filosofía, la Economía o el Derecho. Vemos así, que en cada grado, generalmente, la formación de los docentes en materias de RSC se corresponde con la propia del título en el que se imparte la asignatura.

Llegados a este punto, comprobamos la verificación de nuestras hipótesis:

- Efectivamente, los programas de formación en materia de RSC no se han implantado de forma efectiva en las universidades españolas, como se plantea en la Estrategia española 2014-2020, puesto que todavía hoy más de la mitad de las universidades públicas y casi la mitad de las privadas carecen de formación específica en esta materia.

- Las competencias que se adquieren en los diferentes programas formativos sobre RSC distan entre sí dependiendo del ámbito científico al que pertenece el grado universitario.

Ante esta situación, a modo de reflexión final, podemos concluir que, ciertamente, la formación en materia de RSC en los grados universitarios de las disciplinas científicas vinculadas al ámbito de la gestión empresarial, como Administración y dirección de empresas, Finanzas, Contabilidad, Banca, Seguros, Economía, Marketing, Relaciones laborales, o Derecho, predominantes en las universidades públicas, los contenidos se orientan al cumplimiento de las normas legales y al seguimiento de los indicadores internacionales de calidad en la gestión. De este modo, la formación en materia de responsabilidad social en estos grados se diseña por y para la empresa, siendo el gobierno corporativo el criterio más recurrente en este tipo de enseñanzas. Podríamos decir que en las asignaturas de RSC, en estas titulaciones, el énfasis se deposita sobre la propia organización que la ejerce, esto es, sobre la empresa. Por ello, muy habitualmente se refieren a la RSC como responsabilidad social empresarial, RSE.

Sin embargo, en los grados universitarios de comunicación, en especial en el campo de la Publicidad y Relaciones públicas, la Comunicación audiovisual y el Protocolo, los contenidos de las asignaturas de RSC se orientan a los públicos. Los públicos, los grupos sociales, las personas, son lo importante. Y de este modo se subraya el carácter social de la responsabilidad, independientemente de quien la ejerza. En estas titulaciones, como hemos señalado, la RSC se refiere, sobre todo, a los valores y la relación entre la organización y la sociedad. 
Así, observamos que atendiendo a los cuatro procesos de autorregulación de la gestión corporativa en relación con la responsabilidad social, según Vallaeys, De la Cruz y Sasia (2009), ya citados, en los grados universitarios relacionados con la gestión empresarial y la economía, la RSC se concreta, sobre todo, en el primero de ellos relativo al buen gobierno. Sin embargo, en los grados relacionados con la comunicación, la RSC se orienta hacia los procesos de diálogo y la interlocución con los diferentes públicos de la organización, la gestión de los impactos sociales y medioambientales y una RSC orientada al logro del desarrollo social y medioambiental sostenible basada en una lógica proactiva de implicación en la solución de los problemas sociales del entorno de la organización.

Esta conclusión nos reafirma en el enfoque científico que sustenta nuestra investigación, el de las relaciones públicas excelentes, aquellas en las que la responsabilidad social se convierte en la esencia de las políticas corporativas, con el objetivo de satisfacer las necesidades y los intereses sociales para llegar al entendimiento mutuo entre la organización y sus públicos.

\section{Referencias bibliográficas}

Arias Careaga, S., \& Simón Ruiz, A. (2004). Las estructuras solidarias de las universidades españolas: organización y funcionamiento. Madrid: Universidad Autonoma de Madrid. Disponible en: https://www.upo.es/cms1/export/sites/upo/upsc/ voluntariado/oficina-voluntariado/documentos/libro_estructuras_solidariasxlx.pdf [Consultado el 10/10/2018].

BOE (13/04/2007). "Ley Orgánica 4/2007, de 12 de abril, por la que se modifica la Ley Orgánica 6/2001, de 21 de diciembre, de Universidades”. Boletín Oficial del Estado (89), BOE-A-2007-7786.

BOE (30/10/2007). "Real Decreto 1393/2007, de 29 de octubre, por el que se establece la ordenación de las enseñanzas universitarias oficiales”. Boletín Oficial del Estado (260), BOE-A-2007-18770.

Carroll, A.B. (1991): “The Pyramid of Social Responsibility: Toward the Moral Management of Organizational Stakeholders”. Business Horizons, july-august, pp. 39-48.

Carroll, A.B. (1998). “The four faces of corporate citizenship”. Business and Society Review, Vol. 100 n.1, pp. 1-7.

Consejo Estatal de Responsabilidad Social de las Empresas. CERSE. (2018). Portal de la Responsabilidad Social. Disponible en: http://www.mitramiss.gob.es/es/rse/cerse/index.htm [Consultado el 16/10/2018].

Grupo de Trabajo sobre Educación, Formación y Divulgación de la RSE del CERSE (2010). La responsabilidad social de las empresas (RSE), el desarrollo sostenible y el sistema de educación y Formación. Disponible en: http://www.mitramiss.gob. es/ficheros/rse/documentos/cerse/4_RSE_y_Educacion.pdf [Consultado el 16/10/2018].

De la Calle Maldonado, C. (2011). La Formación de la Responsabilidad Social en la Universidad Católica. Majadahonda, Madrid: Universidad Francisco de Vitoria. Disponible en: http://ddfv.ufv.es/bitstream/handle/10641/294/Calle_Maldonado_139.11.pdf?sequence=1 [Consultado el 27/09/2018]. 
Díaz de Iparraguirre, A. (2008). La Responsabilidad Social de la Universidad en la promoción del capital social para el desarrollo sustentable. Naguanagua.Trabajo final de investigación para optar al título de Magíster en Ciencia Política. Disponible en: http://www.eumed.net/libros-gratis/2008b/402/index.htm [Consultado el 27/09/2018].

Fernández, C.; Delpiano, C. y De Ferari, J.M. (Eds) (2006). Universidad: Construye País. Responsabilidad Social Universitaria. Una manera de ser universidad. Teoría y práctica en la experiencia chilena. Santiago de Chile: Construye País. Disponible en: http://rsuniversitaria.org/web/images/stories/memoria/UCP\%202006.pdf [Consultado el 10/10/2018].

Freeman, R. E. y Reed D.L. (1983). "Stockholders and Stakeholders: A New Perspective on Corporate Governance”. California Management Review; Vol.25 N.3 Spring, pp. 88-106.

García Nieto, Ma Teresa (2012). "Las Ciencias Sociales y la Responsabilidad Social Corporativa”. aDResearch. ESIC Revista Internacional de Investigación en Comunicación n.6, pp.92-111.

González Alcántara, O.J. et al. (2015). La responsabiliad social en las universidades españolas 2014/2015. Alcalá de Henares: IAES.

Grunig, J.E. \& Hunt, T. (1984). Managing Public Relations. New York: Holt, Rinehart and Winston.

Grunig, J.E. (Ed.) (1992). Excellence in Public Relations and Communication Management: Contribution to Effective Organizations. Hillsdale, New Jersey: Lawrence Erlbaum Associates.

López Regalado, M. et al (2015). "Análisis de los Indicadores de la Responsabilidad Social en Empresas de Baja California".. XVIII Congreso AECA. Innovación e internacionalización: factores de éxito para la pyme. Cartagena (España). Disponible en: http://www.aecal.org/pub/on_line/comunicaciones_xviiicongresoaeca/cd/106h.pdf [Consultado el 11/10/2018].

Ministerio de Empleo y Seguridad Social. (2015). Estrategia española de Responsabilidad Social de las empresas. Madrid. Disponible en: http://www.mitramiss.gob.es/ficheros/rse/documentos/eerse/EERSE-Castellano-web.pdf [Consultado el 05/10/2018].

PRME (2007). Principles for Responsible Management Education. Disponible en: http://www.unprme.org/about-prme/ the-six-principles.php [Consultado el 20/10(2018].

UNED (2018). Web corporativa. Responsabilidad Social en la UNED. Disponible en: https://portal.uned.es/portal/page? pageid=93,25080930\&_dad=portal\&_schema=PORTAL [Consultado el 19/10/2018].

Vallaeys, François. (2013). Breve marco teórico de responsabilidad social universitaria. Dsiponible en:

http://www.spring-alfa-pucv.cl/wp-content/uploads/2013/03/breve-marco-teorico-rsu-francois-vallaeys.pdf [Consultado el 12/09/2018].

Vallaeys, F; De la Cruz, C., y Sasia P. M. (2009). Manual de primeros pasos en responsabilización social universitaria: Construyendo ciudadanía en universidades responsables. Disponible en: http://www.cyta.com.ar/biblioteca/bddoc/bdlibros/ rse/334_as_manual_rsu_bid.pdf [Consultado el 12/09/2018]. 